\title{
Alternative lengthening of telomeres and loss of ATRX are frequent events in pleomorphic and dedifferentiated liposarcomas
}

\author{
Jen-Chieh Lee ${ }^{1,2}$, Yung-Ming Jeng ${ }^{1,2}$, Jau-Yu Liau ${ }^{1,2}$, Jia-Huei Tsai ${ }^{1,2}$, Hung-Han Hsu ${ }^{1,2}$ and \\ Ching-Yao Yang ${ }^{3}$
}

${ }^{1}$ Department of Pathology, National Taiwan University Hospital, National Taiwan University College of Medicine, National Taiwan University, Taipei, Taiwan; ${ }^{2}$ Graduate Institute of Pathology, National Taiwan University College of Medicine, National Taiwan University, Taipei, Taiwan and ${ }^{3}$ Department of Surgery, National Taiwan University Hospital, National Taiwan University College of Medicine, National Taiwan University, Taipei, Taiwan

\begin{abstract}
Telomerase activation and alternative lengthening of telomeres are two major mechanisms of telomere length maintenance. Soft tissue sarcomas appear to use the alternative lengthening of telomeres more frequently. Loss of $a$-thalassemia/mental retardation syndrome X-linked (ATRX) or death domain-associated protein 6 (DAXX) expression has been implicated in the pathogenesis of alternative telomere lengthening in pancreatic endocrine neoplasm and glioma. The mechanism leading to the alternative lengthening of telomeres in liposarcoma remains unknown. Whereas alternative telomere lengthening was determined to be an indicator of poor prognosis in liposarcomas as a whole, its prognostic power has not been verified in any subtype of liposarcoma. In this study, we characterized the status of alternative telomere lengthening and expression of ATRX and DAXX in 111 liposarcomas (28 well-differentiated, 52 dedifferentiated, 20 myxoid or round cell, and 11 pleomorphic liposarcomas) by telomere fluorescence in situ hybridization and immunohistochemistry, respectively. Alternative lengthening of telomere was observed in $0 \%(0 / 16)$ of well-differentiated, $30 \%(14 / 46)$ of dedifferentiated, $5 \%(1 / 19)$ of myxoid or round cell, and $80 \%(8 / 10)$ of pleomorphic liposarcomas. Eighteen $(16 \%)$ and one (1\%) tumors were negative for ATRX and DAXX immunostaining, respectively. Remarkably, all cases with loss of either ATRX or DAXX expression had alternative lengthening of telomeres, and $83 \%(19 / 23)$ of tumors that had alternative lengthening of telomeres showed loss of either protein. The correlation between loss of either ATRX or DAXX and alternative telomere lengthening was $100 \%$ in dedifferentiated liposarcoma. The presence of alternative telomere lengthening in dedifferentiated liposarcoma suggested poor overall survival (hazard ratio $=1.954, P=0.077$ ) and was the most significant indicator of short progression-free survival (hazard ratio $=3.119, P=0.003$ ). In conclusion, we found that ATRX loss was the most likely mechanism of alternative telomere lengthening in liposarcoma and alternative telomere lengthening was a prognostic factor of poor outcome in dedifferentiated liposarcoma.

Modern Pathology (2015) 28, 1064-1073; doi:10.1038/modpathol.2015.67; published online 29 May 2015
\end{abstract}

Liposarcoma is one of the most common types of soft tissue sarcoma. Histologically, liposarcoma can be divided into three major groups: well-differentiated or dedifferentiated liposarcoma, myxoid or round cell liposarcoma, and pleomorphic liposarcoma.

Correspondence: Dr C-Y Yang, MD, PhD, Department of Surgery, National Taiwan University Hospital, National Taiwan University College of Medicine, National Taiwan University, No. 7, ChungShan South Road, Taipei 10051, Taiwan.

E-mail: cyang@ntuh.gov.tw

Received 28 February 2015; revised 22 April 2015; accepted 24 April 2015; published online 29 May 2015
Well-differentiated and dedifferentiated liposarcoma is characterized by amplification of the chromosome 12q13-15 region in the form of ring or giant marker chromosomes, with consistent coamplification of $M D M 2$, SAS, HMGA2, and, frequently, CDK4 genes. ${ }^{1,2}$ Myxoid/round cell liposarcoma contains reciprocal gene fusion involving DDIT3 on chromosome 12 with either FUS on chromosome 16 or EWSR1 on chromosome 22.3,4 By contrast, pleomorphic liposarcoma shows complex structural and numerical chromosomal abnormalities. ${ }^{5}$ Dedifferentiated liposarcoma represents an abrupt transition from well-differentiated liposarcoma to a region of 
high-grade, nonlipogenic sarcoma. In addition to the 12q13-15 amplicon, multiple cytogenetic abnormalities have been identified in dedifferentiated liposarcoma, ${ }^{6,7}$ but the mechanism of dedifferentiation remains unclear.

Telomeres are repetitive hexameric TTAGGG DNA sequences at the ends of each chromosome. Telomeres shorten after each round of cell division; therefore, telomere length must be maintained to sustain limitless replicative potential in cancer cells. Two major mechanisms of telomere maintenance in cancer are telomerase activation and alternative lengthening of telomeres. ${ }^{8}$ In most cancers $(85-90 \%)$, telomerase activation is used to maintain the length of telomeres. One crucial mechanism of telomerase activation is mutations in the promoter region of the TERT gene, which encodes the catalytic subunit of the telomerase riboprotein complex. ${ }^{9,10}$ The mutations create new binding motifs for the E-twenty-six family of transcription factors and enhance transcription and protein expression. Alternative lengthening of telomere is a telomeraseindependent pathway of recombination-mediated elongation. ${ }^{11}$ Telomere lengths are maintained through alternative lengthening of telomeres in $10-15 \%$ of cancers. Tumors with alternative lengthening of telomeres are characterized by marked telomere length heterogeneity on Southern blotting and the presence of alternative lengthening of telomere-associated promyelocytic leukemia bodies. ${ }^{12,13}$ Brain tumors, particularly astrocytomas and pediatric glioblastomas, and soft tissue sarcomas appear to use this mechanism frequently. ${ }^{14-16}$

Recently, $61 \%$ of pancreatic neuroendocrine tumors were shown to exhibit alternative lengthening of telomeres, and this phenotype correlated perfectly with the inactivation of either the $\alpha$-thalassemia/mental retardation syndrome X-linked (ATRX) protein or death domain-associated protein 6 (DAXX) protein. ${ }^{17,18}$ Predominant loss of ATRX has been observed in gliomas and cell lines that showed alternative lengthening of telomeres. ${ }^{19,20}$ ATRX and DAXX form a dimer and are crucial for the incorporation of histone 3.3 into telomeres and telomere stability. ${ }^{21-23}$ Although the mechanism has not been delineated, the dysfunction of ATRX or DAXX is thought to cause telomere instability, homologous recombination, and, ultimately, alternative telomere lengthening.

TERT promoter mutation has been identified as a frequent event in myxoid liposarcoma but not in other types of liposarcoma. ${ }^{24}$ Previous studies have reported that alternative lengthening of telomeres was found in $\sim 22-33 \%$ of liposarcomas. ${ }^{15,16,25-28}$ alternative lengthening of telomeres is an indicator of poor clinical outcome in liposarcoma; ${ }^{26,28}$ however, most of these studies have not distinguished histologic subtypes of liposarcoma. In addition, the mechanism of alternative lengthening of telomeres in liposarcoma remains unknown. In this study, we investigated the alternative lengthening of telomeres phenotype in various subtypes of liposarcoma and examined the relationship between alternative lengthening of telomeres and ATRX or DAXX expression in liposarcoma.

\section{Materials and methods}

\section{Tumor Samples and Histological Analysis}

The archives of the Department of Pathology, National Taiwan University Hospital were searched for liposarcoma cases diagnosed from January 1995 to March 2014. Formalin-fixed, paraffin-embedded tissue blocks and slides were retrieved and histological and immunohistochemical sections were reviewed to confirm the diagnoses, which were categorized into well-differentiated liposarcoma, dedifferentiated liposarcoma, myxoid/round cell liposarcoma, and pleomorphic liposarcoma. Histological parameters were revisited, including mitotic counts and necrosis in all types of liposarcoma, and the presence of a round cell component in myxoid/round cell liposarcoma. Twelve dedifferentiated liposarcomas with homologous lipoblastic differentiation had been previously reported and were included in this study. ${ }^{29}$ Tumors were graded according to the scheme of the Fédération Nationale des Centres de Lutte Contre le Cancer (FNCLCC) and staged according to the seventh edition of the TNM staging system of the Union for International Cancer Control. The differentiation scoring as a part of the FNCLCC grading system is not clearly defined in 'low-grade dedifferentiated' liposarcomas. It has been proposed that a mitotic count of at least 5 per 10 high-power fields predicts a poor outcome and is therefore required for diagnosing dedifferentiated liposarcoma, as opposed to a cellular well-differentiated liposarcoma or atypical lipomatous tumor. ${ }^{30}$ Thus, we provisionally allocated a 'modified differentiation score' 1 to cellular nonlipogenic tumors that had $<5$ mitotic figures per 10 high-power fields. The Research Ethics Committee of National Taiwan University Hospital approved this study.

\section{Telomere Fluorescent in Situ Hybridization and Immunohistochemistry}

The protocols for telomere fluorescent in situ hybridization (FISH) and immunohistochemistry were described in our previous report. ${ }^{31}$

\section{Statistical Analysis}

Data analyses were conducted using SPSS Version 19 (IBM Corp., Armonk, NY, USA). Comparisons of categorical variables were performed using the Pearson's $\chi^{2}$ method or the Fisher's exact test. Continuous variables were analyzed using the Student's $t$-test. The survival curves were generated using the Kaplan-Meier method, and the differences 
were calculated using the log-rank test. The Cox proportional hazards regression model was adopted to compare the differences in survival rates. Various cutoff points of mitotic count, size, age, grade, and stage were applied to segregate the cases into two groups for comparison to determine the optimal cutoff points. Disease progression was defined as postoperative recurrence, metastasis, and tumorrelated death. All statistical results were significant at $P<0.05$.

\section{Results}

\section{Clinicopathologic Characteristics}

A total of 111 liposarcoma samples from 88 patients were retrieved and reviewed (Table 1). There were 28 well-differentiated liposarcomas, 52 dedifferentiated liposarcomas, 20 myxoid/round cell liposarcomas, and 11 pleomorphic liposarcomas, including 17 synchronous and 6 metachronous pairs of well-differentiated liposarcomas and dedifferentiated liposarcomas from 22 patients (one patient with a primary tumor harboring both components and a recurrent dedifferentiated liposarcoma). Six myxoid/round cell liposarcomas harbored significant $(>5 \%)$ areas of round cell component and were deemed round cell liposarcomas. Forty-three (39\%) tumors were recurrent, including 11 (39\%) welldifferentiated liposarcomas, 20 (38\%) dedifferentiated liposarcomas, $9(45 \%)$ myxoid/round cell liposarcomas, and $3(27 \%)$ pleomorphic liposarcomas. Two of the recurrent myxoid/round cell liposarcomas were metastatic lesions. Thirteen dedifferentiated liposarcomas were downgraded from conventional FNCLCC grade 2 to 'modified FNCLCC' grade 1. The same group of cases was downstaged from stage II to 'modified' stage I for the same reason.

\section{Telomere FISH}

The FISH results were interpretable in 16 welldifferentiated liposarcomas, 46 dedifferentiated liposarcomas, 19 myxoid/round cell liposarcomas, and 10 pleomorphic liposarcomas. Significant heterogeneity of telomere size, which indicated the presence of alternative lengthening of telomeres, was observed in $30 \%(14 / 46)$ of dedifferentiated liposarcomas, $5 \%(1 / 19)$ of myxoid/round cell liposarcomas, and $80 \%(8 / 10)$ of pleomorphic liposarcomas (Table 2). None of the well-differentiated liposarcomas showed alternative lengthening of telomeres, and 5 were accompanied by synchronous (in one case) or

Table 1 Summary of clinicopathologic features

\begin{tabular}{|c|c|c|c|c|c|}
\hline Tumor type & Well-differentiated & Dedifferentiated & Myxoid/round cell & Pleomorphic & All \\
\hline Number & 28 & 52 & 20 & 11 & 111 \\
\hline Age range (median) & $37-80(67)$ & $29-80(62)$ & $16-71(46)$ & $36-85(66)$ & $16-85(60)$ \\
\hline Sex $(\mathrm{F}: \mathrm{M})$ & $12: 16$ & $20^{\mathrm{a}}: 32$ & $6: 14$ & $3: 8$ & $32: 56$ \\
\hline \multicolumn{6}{|l|}{ Location (\%) } \\
\hline Retroperitoneum & $19(68 \%)$ & $38(73 \%)$ & - & - & $57(51 \%)$ \\
\hline Lower extremity & $2(7 \%)$ & $4(8 \%)$ & $16(80 \%)$ & $8(73 \%)$ & $30(27 \%)$ \\
\hline Pelvis/genitalia & $3(11 \%)$ & $4(8 \%)$ & $1(5 \%)$ & - & $8(7 \%)$ \\
\hline Mediastinum & $2(7 \%)$ & $4(8 \%)$ & - & - & $6(5 \%)$ \\
\hline Limb girdle & $1(4 \%)$ & $1(2 \%)$ & $2(10 \%)$ & $1(9 \%)$ & $5(5 \%)$ \\
\hline Trunk/cavity wall & $1(4 \%)$ & $1(2 \%)$ & $1(5 \%)$ & - & $3(3 \%)$ \\
\hline Upper extremity & - & - & - & $2(18 \%)$ & $2(2 \%)$ \\
\hline Size (cm) (median) & $5.5-42(17)^{\mathrm{b}}$ & $5-42(18)$ & $4.8-22(10)$ & $1.5-30(7)$ & $1.5-42(15)$ \\
\hline \multicolumn{6}{|c|}{ FNCLCC grade (modified ${ }^{\mathrm{C}}$ ) } \\
\hline 1 & 28 & $0(13)$ & 10 & - & 38 \\
\hline 2 & - & $29(16)$ & 9 & 1 & 39 \\
\hline 3 & - & $23(23)$ & 1 & 10 & 34 \\
\hline \multicolumn{6}{|l|}{ Stage $\left(\right.$ modified $^{\mathrm{C}}$ ) } \\
\hline I & 9 & $0(13)$ & 9 & - & 18 \\
\hline II & 12 & $29(16)$ & 7 & 2 & 50 \\
\hline III & 5 & $22(22)$ & 1 & 9 & 37 \\
\hline IV & 1 & $1(1)$ & 3 & - & 5 \\
\hline \multicolumn{6}{|c|}{ Alternative lengthening of telomeres by FISH (\%) } \\
\hline Positive & $0(0 \%)$ & $14(30 \%)$ & $1(5 \%)$ & $8(80 \%)$ & $23(25 \%)$ \\
\hline Negative & $16(100 \%)$ & $32(70 \%)$ & $18(95 \%)$ & $2(20 \%)$ & $68(75 \%)$ \\
\hline Failed & 12 & 6 & 1 & 1 & 20 \\
\hline
\end{tabular}

${ }^{\mathrm{a} O n e}$ female patient had two dedifferentiated liposarcomas included.

${ }^{\mathrm{b}}$ Tumor size is unknown in one well-differentiated liposarcoma.

${ }^{\mathrm{C}}$ Applicable only to dedifferentiated liposarcoma, see text. 
Table 2 The correlation between alternative lengthening of telomeres status and clinicopathologic parameters

\begin{tabular}{|c|c|c|c|c|c|}
\hline \multirow{2}{*}{ Tumor type } & $\begin{array}{c}\text { All } \\
\text { tumors }\end{array}$ & $\begin{array}{c}\text { Excluding } \\
\text { well-differentiated }\end{array}$ & Dedifferentiated & $\begin{array}{l}\text { Myxoid/round } \\
\text { cell }\end{array}$ & Pleomorphic \\
\hline & \multicolumn{5}{|c|}{ Alternative lengthening of telomeres status (positive:negative) } \\
\hline Number & 23:68 & $23: 52$ & $14: 32$ & $1: 18$ & $8: 2$ \\
\hline \multicolumn{6}{|l|}{ Age } \\
\hline Median & $67: 56$ & $67: 54$ & 69:61 & $34: 48( \pm 13)^{\mathrm{a}}$ & $67: 73$ \\
\hline$P$-value & ${ }^{*}<0.001$ & ${ }^{*}<0.001$ & ${ }^{*} 0.003$ & NA & 0.705 \\
\hline \multicolumn{6}{|l|}{ Male gender } \\
\hline Percentage & $65: 62$ & $65: 64$ & $57: 63$ & 100:67 & $75: 50$ \\
\hline$P$-value & 0.767 & 0.884 & 0.732 & 0.485 & 0.490 \\
\hline \multicolumn{6}{|l|}{ Retroperitoneum/ mediastinum } \\
\hline Percentage & $52: 54$ & $52: 48$ & $86: 78$ & $0: 0$ & $0: 0$ \\
\hline$P$-value & 0.853 & 0.744 & 0.551 & NA & NA \\
\hline \multicolumn{6}{|l|}{ Mitotic count (/10 high-power fields) } \\
\hline Median & $15: 4$ & $15: 6$ & $13: 6$ & $22: 5( \pm 3)^{\mathrm{a}}$ & $23: 47$ \\
\hline$P$-value & ${ }^{*} 0.001$ & *0.005 & 0.095 & NA & 0.616 \\
\hline \multicolumn{6}{|l|}{ Necrosis } \\
\hline Percentage & $83: 40$ & $83: 46$ & $86: 59$ & $0: 22$ & $88: 50$ \\
\hline$P$-value & $*<0.001$ & *0.003 & 0.080 & 0.596 & 0.236 \\
\hline \multicolumn{6}{|l|}{ Size } \\
\hline Median & $15: 15$ & $15: 15$ & $16: 18$ & $17: 11( \pm 6)^{\mathrm{a}}$ & $6: 13$ \\
\hline$P$-value & 0.567 & 0.781 & 0.832 & NA & 0.752 \\
\hline \multicolumn{6}{|l|}{ Grade 3} \\
\hline Percentage (modified grades 2 and 3 ) & $83: 15$ & $83: 21$ & $86: 25(66)$ & $0: 6$ & $88: 100$ \\
\hline$P$-value & $*<0.001$ & ${ }^{*}<0.001$ & $*<0.001(0.164)$ & 0.809 & 0.598 \\
\hline \multicolumn{6}{|l|}{ Stages 3 and 4 (modified stages II-IV) } \\
\hline Percentage & $83: 25$ & $83: 25$ & $86: 25$ (66) & 100:17 & $75: 100$ \\
\hline$P$-value & $*<0.001$ & ${ }^{*}<0.001$ & $*<0.001(0.164)$ & *0.047 & 0.429 \\
\hline \multicolumn{6}{|l|}{ Loss of ATRX or DAXX expression } \\
\hline Percentage & 83:0 & 83:0 & 100:0 & $0: 0$ & 63:0 \\
\hline$P$-value & ${ }^{*}<0.001$ & $*<0.001$ & ${ }^{*}<0.001$ & NA & 0.114 \\
\hline
\end{tabular}

Abbreviation: NA: not applicable.

Note: For myxoid/round cell liposarcoma, only one case had alternative lengthening of telomere, thus Student's $t$-test is not performed.

${ }^{\text {a }}$ Standard deviation is shown within parentheses. *Statistically significant.

metachronous (4, including 3 later and one earlier temporally) dedifferentiated liposarcomas that exhibited alternative lengthening of telomeres. Remarkably, of the 12 dedifferentiated liposarcomas with 'homologous lipoblastic differentiation', 7 exhibited alternative lengthening of telomeres. Representative examples are shown in Figures 1-3.

Overall, the presence of alternative lengthening of telomeres was significantly associated with old age, a high stage (stages III and IV), high grade (FNCLCC grade 3), high mitotic count, and the presence of necrosis. Because all of the interpretable FISH results derived from well-differentiated liposarcomas were negative, the overall association with these clinicopathologic parameters might be considerably attributed to the well-differentiated liposarcomas, most of which were low grade, low stage, mitotically inactive, and devoid of tumor necrosis. Therefore, we excluded well-differentiated liposarcomas from the analysis and found alternative lengthening of telomeres was still significantly associated with the aforementioned factors. Further analysis revealed that alternative lengthening of telomeres was significantly associated with a high stage, high FNCLCC grade, and old age in dedifferentiated liposarcoma and with a high stage in myxoid/round cell liposarcoma, whereas 'modified FNCLCC' grade 1 dedifferentiated liposarcoma was not significantly associated with negative results of alternative lengthening of telomeres. Table 2 presents more details.

\section{ATRX and DAXX Immunohistochemistry}

A total of $18(16 \%)$ tumors were negative for ATRX immunostaining, including $13(25 \%)$ dedifferentiated liposarcomas and $5(46 \%)$ pleomorphic liposarcomas. Negative staining for DAXX was observed 

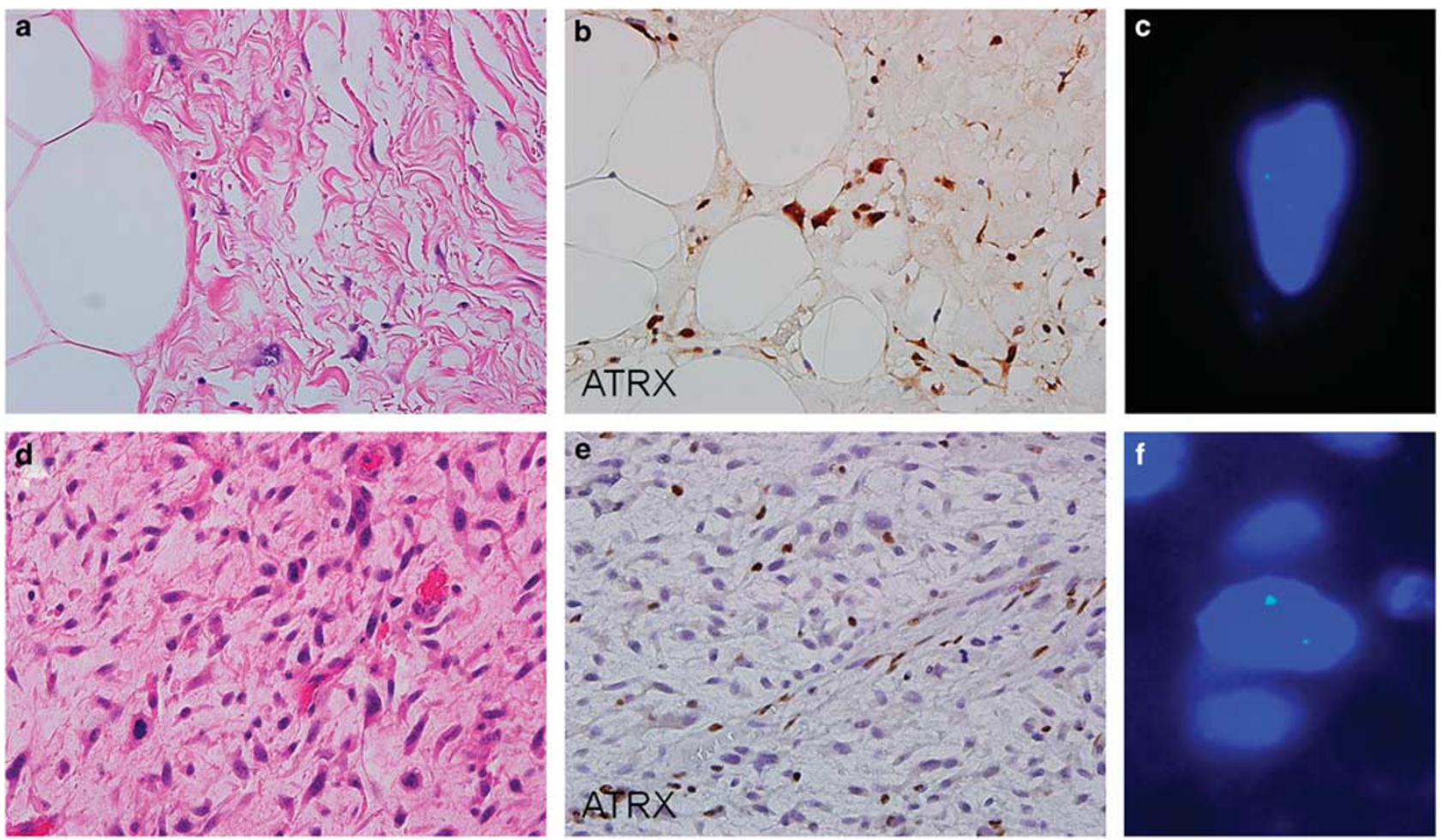

Figure 1 An example of well-differentiated/dedifferentiated liposarcoma with matched histology (hematoxylin-eosin staining), immunohistochemistry, and fluorescent in situ hybridization (FISH). Whereas the well-differentiated component preserves ATRX expression and is negative for alternative telomere lengthening (a-c), the synchronous dedifferentiated focus is negative for ATRX and positive for alternative telomere lengthening (d-f). The endothelial cells and inflammatory cells serve as internal positive control. DAXX is positive in both foci (not shown).

in only $1(1 \%)$ tumor, which was an ATRX-positive dedifferentiated liposarcoma. Remarkably, all cases with loss of either ATRX or DAXX expression had alternative lengthening of telomeres. On the other hand, 4 of the 23 tumors with alternative lengthening of telomeres failed to show loss of either protein, including 3 pleomorphic liposarcomas and one myxoid liposarcoma. The correlation between loss of either ATRX or DAXX and alternative lengthening of telomeres was $100 \%$ in dedifferentiated liposarcoma, including the cases with homologous lipoblastic differentiation. Figures 1-3 shows examples with matched histology, immunostains, and FISH. In summary, the immunostaining results were highly correlated with the phenomenon of alternative lengthening of telomeres $(P<0.001)$ and the correlation was perfect in dedifferentiated liposarcoma.

\section{Survival Analysis}

Because the well-differentiated liposarcoma, myxoid/ round cell liposarcoma, and pleomorphic liposarcoma cases with available clinical follow-up information were either all negative (well-differentiated liposarcoma and myxoid/round cell liposarcoma) or all positive (pleomorphic liposarcoma) for alternative lengthening of telomeres, only dedifferentiated lipo- sarcoma was subjected to survival analysis. According to univariate Cox's proportional hazards analysis, the overall survival was significantly associated only with mitotic count (cutoff points of 5 and 10, but not 20 per 10 high-power fields), modified FNCLCC grade (grade 1 vs higher grades), and modified stage (stage I vs higher stages). Because the mitosis status $(\geq 5$ vs $<5)$, modified FNCLCC grade status (grade 1 vs higher grades), and modified stage status (stage I vs higher stages) were identical in each case, multivariate analysis was not performed. The alternative lengthening of telomeres phenotype suggested adverse overall survival, albeit not statistically significant $(P=0.077)$, but was the factor that most significantly correlated with progression-free survival, compared with other significant factors such as mitotic count (cutoff point of 5 per 10 high-power fields), modified FNCLCC grade (grade 1 vs higher grades), and modified stage (stage I vs higher stages). Table 3 presents the details of survival analysis. The Kaplan-Meier survival plots are shown in Figure 4.

\section{Discussion}

According to cytogenetic features, sarcomas can be generally divided into two groups: (1) sarcomas with specific genetic alterations and typically simple 

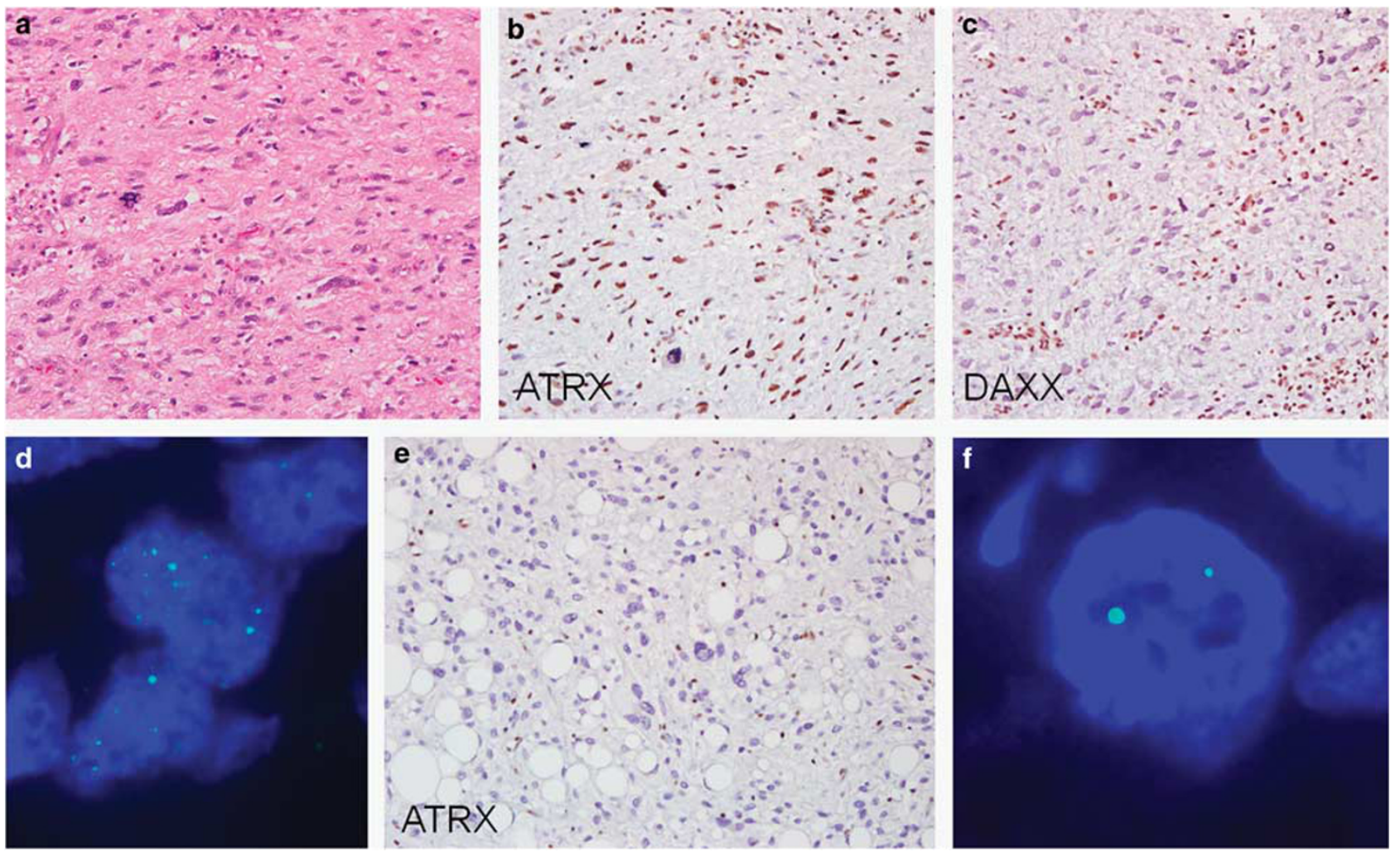

Figure 2 Another dedifferentiated liposarcoma is ATRX-positive, DAXX-negative, and positive for alternative telomere lengthening (a-d). The inflammatory cells serve as positive control for the immunostains. (e and f) A dedifferentiated liposarcoma with prominent homologous lipoblastic differentiation exhibits loss of ATRX (in both lipoblasts and non-lipoblastic cells) and a phenotype of alternative telomere lengthening.

karyotypes and (2) sarcomas with more complex karyotypes. $^{32}$ The first group includes sarcomas with reciprocal chromosomal translocations and specific oncogenic mutations, and the other include most adult sarcomas, which contain multiple genetic abnormalities, including chromosomal numerical changes, translocations, gene amplifications, and large deletions. Although sharing the feature of lipogenic differentiation, different types of liposarcoma are distinct in chromosomal and genetic aberrations. Myxoid/round cell liposarcoma is characterized by recurrent gene fusion involving DDIT3 and belongs to sarcomas of the first group. Pleomorphic liposarcoma and dedifferentiated liposarcoma show more complex chromosomal abnormalities and thus belong to the second group. Although well-differentiated liposarcoma shares with dedifferentiated liposarcoma the presence of supernumerary ring or giant marker chromosomes derived from chromosome 12, this abnormality sometimes exists as the sole chromosomal change in well-differentiated liposarcoma. ${ }^{33}$ Overall, welldifferentiated liposarcoma has a simpler karyotype compared with that of dedifferentiated liposarcoma and, therefore, might be categorized into the first group.

The alternative lengthening of telomeres phenotype is more commonly observed in sarcomas and gliomas than in carcinomas. ${ }^{15}$ Montgomery et $a l^{34}$ showed that telomere lengths were either similar to or reduced compared with surrounding nonneoplastic tissues in sarcomas with specific translocations. By contrast, telomeres were often found to be markedly lengthened and heterogeneous in sarcomas lacking specific translocations. ${ }^{34}$ Because marked telomere length heterogeneity is the cardinal feature of alternative lengthening of telomeres, these results suggested that sarcomas with complex karyotypes tend to use alternative lengthening of telomeres as their telomere maintenance mechanism. In line with this theory, we observed frequent alternative lengthening of telomeres in pleomorphic liposarcoma and dedifferentiated liposarcoma; in contrast, only one myxoid/round cell liposarcoma and no well-differentiated liposarcoma showed the alternative lengthening of telomeres phenotype. The other major telomere maintenance mechanism is telomerase activation. One of the most crucial mechanisms of telomerase activation is mutation in the promoter region of the TERT gene. ${ }^{9}$ Recently, Koelsche et $a l^{24}$ determined that the TERT promoter is frequently mutated in myxoid liposarcoma (74\%) but not in other types of sarcoma, including pleomorphic liposarcoma and dedifferentiated liposarcoma. Therefore, whereas the predominant telomere maintenance mechanism for myxoid liposarcoma is 

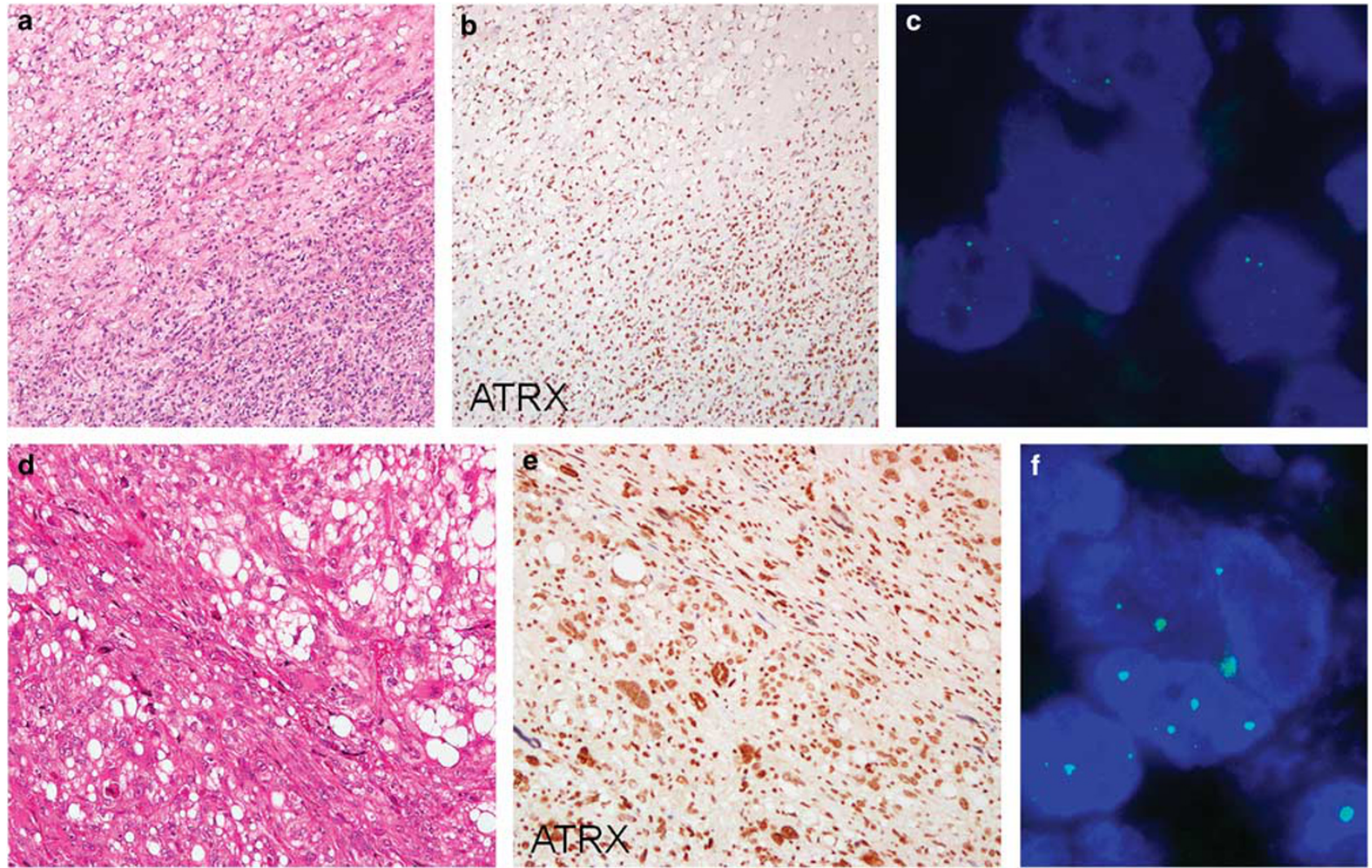

Figure 3 (a-c) A mixed myxoid/round cell liposarcoma is positive for both ATRX and DAXX (not shown) and negative for alternative telomere lengthening in both myxoid (upper left field in a and b) and round cell (lower right field) components. (d-f) A pleomorphic liposarcoma, albeit positive for both ATRX and DAXX, shows prominent alternative lengthening of telomeres.

telomerase activation, that for pleomorphic liposarcoma and dedifferentiated liposarcoma is alternative lengthening of telomeres. Both telomere maintenance mechanisms seemed infrequent in well-differentiated liposarcoma, which may explain the limited agressiveness of well-differentiated liposarcoma. ${ }^{26}$

Most previous studies addressing the role of alternative lengthening of telomeres in liposarcomas have not differentiated subtypes of liposarcoma. In particular, pleomorphic liposarcoma has not been systemically analyzed separately. The only study that included subtype information indicated that alternative lengthening of telomeres was prevalent in dedifferentiated and grade 3 liposarcomas, which was similar to the result obtained in our study. ${ }^{26}$ The major difference between the two studies is that they detected alternative lengthening of telomeres in $14 \%$ of well-differentiated liposarcomas and $18 \%$ of myxoid/round cell liposarcomas by using an assay to characterize alternative lengthening of telomereassociated promyelocytic leukemia bodies, whereas we used telomeric FISH and determined that alternative lengthening of telomeres was almost limited to dedifferentiated liposarcoma and pleomorphic liposarcoma. The discrepancy might have stemmed
Table 3 Correlation of survival of dedifferentiated liposarcoma patients and clinicopathologic features

\begin{tabular}{|c|c|c|c|c|}
\hline \multirow{2}{*}{ Factors } & \multicolumn{2}{|c|}{$\begin{array}{l}\text { Overall } \\
\text { survival }\end{array}$} & \multicolumn{2}{|c|}{$\begin{array}{l}\text { Progression- } \\
\text { free survival }\end{array}$} \\
\hline & $H R$ & P-value & $H R$ & $\mathrm{P}$-value \\
\hline $\begin{array}{l}\text { Alternative lengthening of } \\
\text { telomeres }\end{array}$ & 1.954 & 0.077 & 3.119 & ${ }^{*} 0.003$ \\
\hline Mitoses $(\geq 5$ vs <5) & 4.363 & *0.007 & 2.689 & ${ }^{*} 0.017$ \\
\hline Mitoses $(\geq 10$ vs $<10)$ & 2.223 & *0.034 & 1.699 & 0.122 \\
\hline Mitoses $(\geq 20$ vs $<20)$ & 1.286 & 0.561 & 0.998 & 0.997 \\
\hline Grade (grade 3 vs grades 1 and 2) & 1.743 & 0.132 & 1.395 & 0.329 \\
\hline $\begin{array}{l}\text { Modified grade (grades } 2 \text { and } \\
3 \text { vs grade } 1 \text { ) }\end{array}$ & 4.363 & $* 0.007$ & 2.689 & * 0.017 \\
\hline $\begin{array}{l}\text { Stage (stages III and IV vs } \\
\text { stages I and II) }\end{array}$ & 1.743 & 0.132 & 1.395 & 0.329 \\
\hline $\begin{array}{l}\text { Modified stage (stages II-IV vs } \\
\text { stage I) }\end{array}$ & 4.363 & ${ }^{*} \mathbf{0 . 0 0 7}$ & 2.689 & ${ }^{*} 0.017$ \\
\hline Age $(>50$ vs $\leq 50)$ & 2.050 & 0.182 & 1.688 & 0.243 \\
\hline Age $(>60$ vs $\leq 60)$ & 2.108 & 0.073 & 1.317 & 0.431 \\
\hline Sex (male vs female) & 1.067 & 0.864 & 0.808 & 0.545 \\
\hline Necrosis & 1.479 & 0.345 & 1.144 & 0.706 \\
\hline Size $(>10$ vs $\leq 10)$ & 1.255 & 0.621 & 1.225 & 0.650 \\
\hline Size $(>15$ vs $\leq 15)$ & 0.699 & 0.332 & 0.734 & 0.360 \\
\hline Size $(>20$ vs $\leq 20)$ & 0.618 & 0.253 & 0.521 & 0.082 \\
\hline $\begin{array}{l}\text { Site (retroperitoneum/ } \\
\text { mediastinum vs others) }\end{array}$ & 1.434 & 0.463 & 2.570 & 0.053 \\
\hline
\end{tabular}

The bold and asterisks denote statistical significance. 

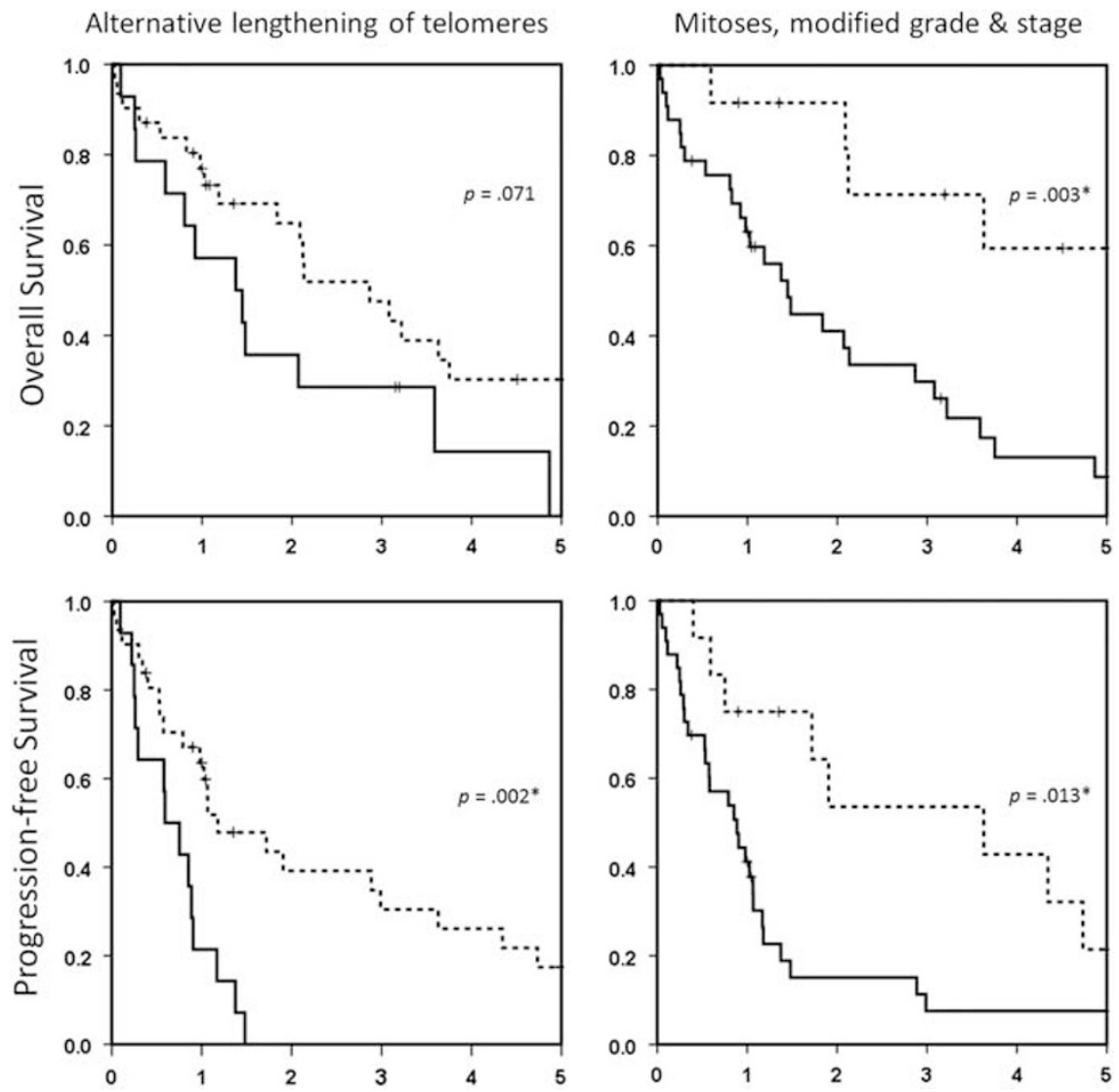

Figure 4 Overall survival (upper panel) and progression-free survival (lower panel) illustrated with Kaplan-Meier plots. Dotted lines indicate cases with negative results of alternative lengthening of telomeres, $<5$ mitotic figures/10 high-power fields, modified grade 1 , or modified stage I; solid lines indicate cases with positive results of alternative lengthening of telomeres, $\geq 5$ mitotic figures/10 high-power fields, modified grade 2 or 3, or modified stages II-IV. The patient groups so dichotomized according to the mitotic count, modified grade, and modified stage overlap completely with one another; therefore only two populations (lower mitotic count/grade/stage vs higher mitotic count/grade/stage) are shown in the right panel.

from different methods used to characterize alternative lengthening of telomeres. A positive telomeric focus is defined as one with the intensity being $>10$-fold that of average telomeres. ${ }^{15}$ Admittedly, the interpretation of signal intensities may be subjective, particularly for signals with borderline intensities. Furthermore, signal intensities are susceptible to tissue or technical variations. For instance, we observed a much higher failure rate of FISH performed on well-differentiated liposarcoma than on other tumor types $(43: 10 \%)$. On the other hand, the promyelocytic leukemia body assay additionally requires such a high-intensity telomeric focus be localized within a promyelocytic leukemia protein body, ${ }^{26}$ thus possibly facilitating the interpretation of borderline foci. The different thresholds for the fraction of tumor of cells ( 0.5 vs $1 \%$ ) harboring the positive foci, as well as the different ethnic groups included, might also account in part for the discrepancy.

The underlying mechanism of alternative lengthening of telomeres in liposarcomas has yet to be elucidated. Pancreatic endocrine tumors with the alternative lengthening of telomeres phenotype were recently found to be $100 \%$ correlated with the inactivation of either ATRX or DAXX. ${ }^{17,18}$ Therefore, loss of the ATRX/DAXX dimer was suggested to be a critical event in tumors with alternative lengthening of telomeres. ${ }^{18}$ In addition, mutation and loss of ATRX, but not DAXX, was identified in gliomas and correlated with alternative lengthening of telomeres in most cases. ${ }^{19}$ In another study, loss of ATRX expression was observed in $90 \%$ of cell lines with alternative lengthening of telomeres, suggesting that the inactivation of ATRX is a major mechanism in tumors with alternative lengthening of telomeres. ${ }^{20}$ Our study revealed for the first time that ATRX expression was lost in liposarcomas. In addition, we demonstrated that the loss of ATRX was highly correlated with alternative lengthening of telomeres in liposarcomas. By contrast, DAXX expression was preserved in all but one case. Therefore, liposarcoma was similar to glioma regarding the frequent inactivation of ATRX but not DAXX in the induction of the alternative lengthening of telomeres phenotype. Nonetheless, ATRX and DAXX were retained in four liposarcomas with alternative lengthening of telomeres, indicating that other mechanisms can lead to alternative lengthening of telomeres in liposarcomas. 
Dedifferentiation is a process in which a welldifferentiated liposarcoma transforms into a metastasis-capable dedifferentiated liposarcoma, which may cause more serious clinical problems. The mechanism of dedifferentiation of well-differentiated liposarcoma remains unclear. In our study, 22 patients had paired samples of well-differentiated and dedifferentiated liposarcomas and 5 of them showed alternative lengthening of telomeres and loss of ATRX only in the dedifferentiated part. Alternative lengthening of telomeres and loss of ATRX is associated with genome instability in sarcoma cell lines. ${ }^{20}$ Cells with the alternative lengthening of telomeres phenotype have marked heterogeneity in telomere length, and some chromosomes may even lack telomeric sequences. These unprotected telomeres may lead to chromosome fusion and ultimately genome instability and high-grade transformation. Therefore, the emergence of clones with alternative lengthening of telomeres may conceivably contribute to dedifferentiation in some cases. However, because most dedifferentiated liposarcomas lacked alternative lengthening of telomeres, other mechanisms responsible for dedifferentiation should be sought. Remarkably, although a previous study suggested the stability of alternative lengthening of telomeres status throughout disease evolution in liposarcomas, ${ }^{27}$ one patient in our series had a primary dedifferentiated liposarcoma lacking alternative lengthening of telomeres that appeared later in the recurrent dedifferentiated liposarcoma. Collectively, our findings indicate that conversion of alternative lengthening of telomeres status exists during liposarcoma progression, often in the form of dedifferentiation. Additional studies focusing on cases with multiple metachronous tumors may be required to further address this topic. Previous reports have indicated that alternative lengthening of telomeres is an indicator of poor clinical outcome in liposarcomas as a whole. ${ }^{26,28}$ This is not unexpected because dedifferentiated liposarcoma and pleomorphic liposarcoma generally have poorer prognoses than well-differentiated liposarcoma and myxoid liposarcoma do. Nonetheless, whether alternative lengthening of telomeres status remains prognostically significant in any single histological type of liposarcoma has not been corroborated. We determined that alternative lengthening of telomeres was the most significant factor that predicted a short progression-free survival in dedifferentiated liposarcoma. According to this observation, dedifferentiated liposarcomas with alternative lengthening of telomeres were often high-grade tumors with higher mitotic count and necrosis, which are histological features that generally suggest aggressive tumor behavior. Remarkably, inhibition of protein kinase ATR has been shown to selectively cause apoptosis of cells with alternative lengthening of telomeres, a discovery that may endow the current findings with therapeutic implications. ${ }^{35}$ In addition, the finding that a mitotic count of 5 in 10 high-power fields effectively dichotomized the dedifferentiated liposarcoma cases into two distinct prognostic groups supports the notion that at least five mitotic figures per 10 high-power fields are required for diagnosing conventional dedifferentiated liposarcoma ${ }^{30}$ and indicates that the concept of low-grade dedifferentiated liposarcomas might warrant further clarification factoring in mitosis.

The molecular mechanism for the loss of ATRX expression in liposarcoma is unknown. According to the provisional data from The Cancer Genome Atlas (http://cancergenome.nih.gov/), 12.5\% (7/56) of dedifferentiated liposarcomas harbored homozygous ATRX deletion, which might partly account for the loss of ATRX expression. However, because our data showed ATRX loss in 25\% of dedifferentiated liposarcomas, other mechanisms such as mutations and epigenetic silencing might also be implicated. Further investigation must be conducted to solve this problem.

Our study has several limitations. First, the case numbers of myxoid/round cell liposarcoma and pleomorphic liposarcoma were relatively small and, therefore, the prognostic power of alternative lengthening of telomeres could not be analyzed. Second, the FISH assay frequently failed in well-differentiated liposarcomas so that the results might not be representative enough. Third, many of the dedifferentiated liposarcomas had preceding or concurrent welldifferentiated components, which were ignored in the survival analysis for the purpose of simplicity. The influence contributed by the well-differentiated components might thus be underestimated.

In conclusion, we found a high frequency of alternative lengthening of telomeres in pleomorphic liposarcoma and dedifferentiated liposarcoma but not in myxoid/round cell liposarcoma or welldifferentiated liposarcoma. Alternative lengthening of telomeres correlated with poor prognosis in dedifferentiated liposarcoma. Loss of ATRX expression is likely an underlying mechanism for alternative lengthening of telomeres in liposarcoma. In addition, the role for alternative lengthening of telomeres in dedifferentiation of well-differentiated liposarcoma warrants further investigation.

\section{Acknowledgments}

This study was supported by grants provided by the National Science Council, Taiwan to CYY (NSC 97-2314-B-002-026-MY3，101-2320-B-002-044-MY3) and by the National Taiwan University Hospital to CYY (NTUH 104-S2644) and to JCL (NTUH 104-S2789). We thank the staff of the Department of Medical Research, National Taiwan University Hospital for the support.

\section{Disclosure/conflict of interest}

The authors declare no conflict of interest. 


\section{References}

1 Dei Tos AP, Doglioni C, Piccinin S et al. Coordinated expression and amplification of the MDM2, CDK4, and HMGI-C genes in atypical lipomatous tumours. J Pathol 2000;190:531-536.

2 Italiano A, Bianchini L, Keslair $\mathrm{F}$ et al. HMGA2 is the partner of MDM2 in well-differentiated and dedifferentiated liposarcomas whereas CDK4 belongs to a distinct inconsistent amplicon. Int J Cancer 2008;122: 2233-2241.

3 Aman P, Ron D, Mandahl N et al. Rearrangement of the transcription factor gene CHOP in myxoid liposarcomas with t(12;16)(q13;p11). Genes Chromosomes Cancer 1992;5:278-285.

4 Crozat A, Aman P, Mandahl N, Ron D. Fusion of CHOP to a novel RNA-binding protein in human myxoid liposarcoma. Nature 1993;363:640-644.

5 Mertens F, Fletcher CD, Dal Cin P et al. Cytogenetic analysis of 46 pleomorphic soft tissue sarcomas and correlation with morphologic and clinical features: a report of the CHAMP Study Group. Chromosomes and MorPhology. Genes Chromosomes Cancer 1998;22:16-25.

6 Rieker RJ, Joos S, Bartsch C et al. Distinct chromosomal imbalances in pleomorphic and in high-grade dedifferentiated liposarcomas. Int J Cancer 2002;99:68-73.

7 Hostein I, Coindre JM, Derre J et al. Comparative genomic hybridization study of paraffin-embedded dedifferentiated liposarcoma fixed with Holland Bouin's fluid. Diagn Mol Pathol 2003;12:166-173.

8 Gunes C, Rudolph KL. The role of telomeres in stem cells and cancer. Cell 2013;152:390-393.

9 Horn S, Figl A, Rachakonda PS et al. TERT promoter mutations in familial and sporadic melanoma. Science 2013;339:959-961.

10 Huang FW, Hodis E, Xu MJ et al. Highly recurrent TERT promoter mutations in human melanoma. Science 2013;339:957-959.

11 Draskovic I, Londono Vallejo A. Telomere recombination and alternative telomere lengthening mechanisms. Front Biosci (Landmark Ed) 2013;18:1-20.

12 Royle NJ, Foxon J, Jeyapalan JN et al. Telomere length maintenance-an ALTernative mechanism. Cytogenet Genome Res 2008;122:281-291.

13 Cesare AJ, Reddel RR. Alternative lengthening of telomeres: models, mechanisms and implications. Nat Rev Genet 2010;11:319-330.

14 Henson JD, Reddel RR. Assaying and investigating alternative lengthening of telomeres activity in human cells and cancers. FEBS Lett 2010;584:3800-3811.

15 Heaphy CM, Subhawong AP, Hong SM et al. Prevalence of the alternative lengthening of telomeres telomere maintenance mechanism in human cancer subtypes. Am J Pathol 2011;179:1608-1615.

16 Henson JD, Hannay JA, McCarthy SW et al. A robust assay for alternative lengthening of telomeres in tumors shows the significance of alternative lengthening of telomeres in sarcomas and astrocytomas. Clin Cancer Res 2005;11:217-225.

17 Jiao Y, Shi C, Edil BH et al. DAXX/ATRX, MEN1, and mTOR pathway genes are frequently altered in pancreatic neuroendocrine tumors. Science 2011;331: 1199-1203.

18 Heaphy CM, de Wilde RF, Jiao Y et al. Altered telomeres in tumors with ATRX and DAXX mutations. Science 2011;333:425.
19 Abedalthagafi M, Phillips JJ, Kim GE et al. The alternative lengthening of telomere phenotype is significantly associated with loss of ATRX expression in high-grade pediatric and adult astrocytomas: a multiinstitutional study of 214 astrocytomas. Mod Pathol 2013;26:1425-1432.

20 Lovejoy CA, Li W, Reisenweber S et al. Loss of ATRX, genome instability, and an altered DNA damage response are hallmarks of the alternative lengthening of telomeres pathway. PLoS Genet 2012;8: e1002772.

21 Drane P, Ouararhni K, Depaux A et al. The deathassociated protein DAXX is a novel histone chaperone involved in the replication-independent deposition of H3.3. Genes Dev 2010;24:1253-1265.

22 Goldberg AD, Banaszynski LA, Noh KM et al. Distinct factors control histone variant H3.3 localization at specific genomic regions. Cell 2010;140:678-691.

23 Wong LH, McGhie JD, Sim M et al. ATRX interacts with H3.3 in maintaining telomere structural integrity in pluripotent embryonic stem cells. Genome Res 2010;20:351-360.

24 Koelsche C, Renner M, Hartmann W et al. TERT promoter hotspot mutations are recurrent in myxoid liposarcomas but rare in other soft tissue sarcoma entities. J Exp Clin Cancer Res 2014;33:33.

25 Johnson JE, Varkonyi RJ, Schwalm J et al. Multiple mechanisms of telomere maintenance exist in liposarcomas. Clin Cancer Res 2005;11:5347-5355.

26 Costa A, Daidone MG, Daprai L et al. Telomere maintenance mechanisms in liposarcomas: association with histologic subtypes and disease progression. Cancer Res 2006;66:8918-8924.

27 Venturini L, Erdas R, Costa A et al. ALT-associated promyelocytic leukaemia body (APB) detection as a reproducible tool to assess alternative lengthening of telomere stability in liposarcomas. J Pathol 2008;214: 410-414.

28 Venturini L, Motta R, Gronchi A et al. Prognostic relevance of ALT-associated markers in liposarcoma: a comparative analysis. BMC Cancer 2010;10:254.

29 Liau JY, Lee JC, Wu CT et al. Dedifferentiated liposarcoma with homologous lipoblastic differentiation: expanding the spectrum to include low-grade tumours. Histopathology 2013;62:702-710.

30 Evans HL. Atypical lipomatous tumor, its variants, and its combined forms: a study of 61 cases, with a minimum follow-up of 10 years. Am J Surg Pathol 2007;31:1-14.

31 Liau JY, Tsai JH, Jeng YM et al. Leiomyosarcoma With Alternative lengthening of telomeres is associated with aggressive histologic features, loss of ATRX expression, and poor clinical outcome. Am J Surg Pathol 2015;39: 236-244.

32 Antonescu CR. The role of genetic testing in soft tissue sarcoma. Histopathology 2006;48:13-21.

33 Sandberg AA. Updates on the cytogenetics and molecular genetics of bone and soft tissue tumors: liposarcoma. Cancer Genet Cytogenet 2004;155:1-24.

34 Montgomery E, Argani P, Hicks JL et al. Telomere lengths of translocation-associated and nontranslocationassociated sarcomas differ dramatically. Am J Pathol 2004;164:1523-1529.

35 Flynn RL, Cox KE, Jeitany $\mathrm{M}$ et al. Alternative lengthening of telomeres renders cancer cells hypersensitive to ATR inhibitors. Science 2015;347:273-277. 